\title{
Impact of top-Higgs couplings on Di-Higgs production at future colliders
}

\author{
Ning Liu, ${ }^{a}$ Songlin $\mathrm{Hu}^{a}$ Bingfang Yang ${ }^{a, b}$ and Jinzhong $\mathrm{Han}^{c}$ \\ ${ }^{a}$ College of Physics \& Electronic Engineering, Henan Normal University, \\ Xinxiang 45300\%, China \\ ${ }^{b}$ School of Materials Science and Engineering, Henan Polytechnic University, \\ Jiaozuo 454000, China \\ ${ }^{c}$ School of Physics and Electromechnical Engineering, Zhoukou Normal University, \\ Zhoukou, 466001, China \\ E-mail: wlln@mail.ustc.edu.cn, songlinhu@gmail.com, \\ yangbingfang@gmail.com, hanjinzhongxx@gmail.com
}

ABSTRACT: Measuring the Higgs-self coupling is one of the most crucial goals of the future colliders, such as the LHC Run-II and the ILC-based photon collider. Since the new physics can affects the di-Higgs production not only from the Higgs self-coupling but also from the top-Higgs coupling, we investigate the di-Higgs production in the presence of the non-standard top-Higgs coupling at the LHC and ILC-based photon collider given the recent Higgs data. Due to the changed interference behaviors of the top quark loops with itself or $W$ boson loops, we find that the cross section of di-Higgs production at the LHC$14 \mathrm{TeV}$ and ILC-500 GeV can be respectively enhanced up to nearly 3 and 2 times the SM predictions within $2 \sigma$ Higgs data allowed parameter region.

Keywords: Beyond Standard Model, Heavy Quark Physics

ARXIV EPRINT: 1408.4191 


\section{Contents}

1 Introduction 1

2 Top-Higgs interaction and calculations $\quad 2$

$2.1 g g \rightarrow h h \quad 3$

$2.2 \gamma \gamma \rightarrow h h \quad 4$

3 numerical results and discussions 5

$3.1 \quad g g \rightarrow h h \quad 6$

$\begin{array}{lll}3.2 & \text { ILC-based photon collider } & 7\end{array}$

4 Conclusions $\quad 8$

\section{Introduction}

In 2012, the ATLAS and CMS collaborations jointly announced that a bosonic resonance with a mass around $125 \mathrm{GeV}$ was found at the LHC [1,2]. So far, most measurements of its properties are compatible with the predictions of the Higgs boson in the Standard Model (SM) [3-6]. However, due to the current limited statistics, the Higgs couplings with top quarks and with itself are still vacant and remain to be verified at the future colliders.

In the SM, the couplings of fermions to the Higgs boson are proportional to their masses. Due to the large mass, top quark has the strongest coupling to the Higgs boson and is speculated as a sensitive probe to the new flavor dynamics beyond the SM. As a direct way to test the top-Higgs coupling, the associated production of the top pair with Higgs boson has been widely studied at the LHC [7-22]. Besides, the search for a single top associated production with the Higgs boson was proposed to determine the sign of the top-Higgs coupling at the LHC [23-39]. On the other hand, the top-Higgs coupling also plays a vital role in other processes involving the Higgs boson through the quantum effects, such as the di-Higgs production [40-47]. This makes the top-Higgs coupling inevitably entangled with the Higgs self-coupling and affects the measurement of the Higgs self-coupling at the LHC.

In the renormalizable Lagrangian of the SM, only the quartic Higgs coupling $\lambda\left(\phi^{\dagger} \phi\right)^{2}$ allowed by the electroweak gauge symmetry can generate the Higgs self-coupling. The measurement of the Higgs self-coupling is essential to reconstruct the Higgs potential and understand the electroweak symmetry breaking (EWSB) mechanism. In some extensions of the SM, the self-coupling can be significantly distorted by the loop corrections and become sensitive to the new physics [48-57]. Besides, a large deviation in the self-coupling may be a direct evidence for strong first-order electroweak phase transition in the early universe $[58,59]$. At the LHC, the di-Higgs production is the only way to measure the Higgs self-coupling and is dominated by the gluon-gluon fusion mechanism, which has 
been widely studied in recent years (see examples in SM after $125 \mathrm{GeV}$ Higgs boson [6064]; see examples in MSSM and NMSSM after $125 \mathrm{GeV}$ Higgs boson, [65-69]; see examples in $2 \mathrm{HDM}$ after $125 \mathrm{GeV}$ Higgs boson, [70-73]; see examples in other models after $125 \mathrm{GeV}$ Higgs boson, [74-85]). Among various decay channels, although the $4 b$ final state has the largest fraction, the rare process $h h \rightarrow b \bar{b} \gamma \gamma$ is expected to have the most promising sensitivity due to the low QCD backgrounds at the LHC [86-93].

In this work, we will investigate the effect of non-standard top-Higgs coupling in the di-Higgs production at the LHC and ILC-based photon collider under the current Higgs data constraints. Whenever examining the Higgs self-coupling at the LHC, one should keep in mind that, the main process $g g \rightarrow h h$ can also be triggered by the top-Higgs coupling itself through the box diagrams. These irrelevant processes lead to the strong cancellation with those involving the self-coupling in the SM, which makes the cross section of diHiggs production nearly $10^{3}$ times smaller than the single Higgs production at the LHC. So, the top-Higgs coupling will affect the extraction of the Higgs self-coupling from the measurement of di-Higgs production at the LHC [94].

Given the limited precision of the LHC, an $e^{+} e^{-}$collider is crucial to scrutinize the detailed properties of the Higgs boson that might uncover the new physics beyond the SM [95]. In addition to the $e^{+} e^{-}$collisions, high energy photon-photon collisions can be achieved at the ILC by converting the energetic electron beam to a photon beam through the backward Compton scattering [96]. Similar to the process $g g \rightarrow h h$ at the LHC, $\gamma \gamma \rightarrow h h$ also occurs at one-loop level. The measurements of the Higgs self-coupling at the photon collider were discussed in refs. [97-101], where the complementarity of the photon and $e^{+} e^{-}$collider was emphasized. Recently, an extensive study of the feasibility of the diHiggs production with a parameter set of the ILC-based photon collider was reanalysed in ref. [102], which concluded that that the channel $\gamma \gamma \rightarrow h h \rightarrow b \bar{b} b \bar{b}$ process can be observed with a statistical significance of about $5 \sigma$ for the integrated luminosity corresponding to 5 years running of the photon collider. Therefore, the photon collider provide an ideal place to study the new physics effect in the di-Higgs production.

The structure of this paper is organized as follows. In section 2 , we will briefly introduce the non-standard top-Higgs interaction and set up the calculations. In section 3, we present the numerical results and discuss the effects of non-standard top-Higgs coupling in the di-Higgs production at the LHC and ILC-based photon collider. Finally, we draw our conclusions in section 4 .

\section{Top-Higgs interaction and calculations}

In the SM the top-Higgs interaction can be written as:

$$
\mathcal{L}_{t \bar{t} h}^{\mathrm{SM}}=-y_{t_{\mathrm{SM}}} \bar{Q}_{3 L} t_{R} \tilde{\phi}+\text { h.c. }
$$

with

$$
y_{t_{\mathrm{SM}}}=\sqrt{2} m_{t} / v .
$$

where $Q_{3 L}$ is the third generation SM quark doublet, $\phi$ is the Higgs doublet, $\tilde{\phi}_{i}=\epsilon_{i j} \phi_{j}$, and Higgs field vacuum expectation value (vev) $v \approx 246 \mathrm{GeV}$. However, in some new physics 
models, the top-Higgs interaction can be different from the above SM prediction. These new physics effects on $t \bar{t} h$ coupling can be model-independently parameterized by a gauge invariant dimension-six operator [103]. For example, the term

$$
\mathcal{L}_{t \bar{t} h}^{6}=-\frac{C_{u \phi}^{33}}{\Lambda^{2}}\left(\phi^{\dagger} \phi\right)\left(\bar{Q}_{3 L} t_{R} \tilde{\phi}\right)+\text { h.c.. }
$$

Here we should note that the eq. (2.3) does correct the top quark mass $m_{t}$ by $\frac{v^{3}}{2 \Lambda^{2}}\left[\operatorname{Re} C_{u \phi}^{33}+\right.$ $\operatorname{Im} C_{u \phi}^{33} \gamma_{5}$, which has to be reabsorbed into the physical observable $m_{t}$ in eq. (2.2). With this in mind, after the EWSB, we can have a general $t \bar{t} h$ interaction including the SM top-Higgs couplings and corrections from the dimension-six operator as following,

$$
\mathcal{L}_{t \bar{t} h}=-\frac{y_{t}}{\sqrt{2}} \bar{t}\left(\cos \theta+i \sin \theta \gamma^{5}\right) t h,
$$

with

$$
y_{t} \cos \theta=y_{t_{\mathrm{SM}}}+\frac{v^{2}}{\Lambda^{2}} \operatorname{Re} C_{u \phi}^{33}, \quad y_{t} \sin \theta=\frac{v^{2}}{\Lambda^{2}} \operatorname{Im} C_{u \phi}^{33} .
$$

where $y_{t}$ takes the SM value $y_{t_{\mathrm{SM}}}$ when $\operatorname{Re} C_{u \phi}^{33}=\operatorname{Im} C_{u \phi}^{33}=0$. For convenience, we define two reduced couplings: $c_{t}=y_{t} \cos \theta / y_{t_{\mathrm{SM}}}$ and $\tilde{c}_{t}=y_{t} \sin \theta / y_{t_{\mathrm{SM}}}$ in the following calculations. Although the CP-violating interaction can contribute to the electric dipole moment (EDM), the bounds on the coupling $\tilde{c}_{t}$ rely on the assumption of Higgs couplings to other light fermions $[104,105]$. Given that these couplings are generally unobservable at the LHC, we do not impose EDM constraints in this study. For other low-energy physics constraints, such as $B_{s}-\bar{B}_{s}$ and $B \rightarrow X_{s} \gamma$, they are still too weak [106]. The most relevant indirect constraint is from the current Higgs data since the non-standard topHiggs interaction can change the production rate of $g g \rightarrow h$ and decay width of $h \rightarrow \gamma \gamma$ through the loop effect. The signal strengthes $\mu_{i}$ can be parameterized through the reduced couplings as following [106],

$$
\begin{aligned}
& \mu_{h g g} \simeq c_{t}^{2}+2.6 \tilde{c}_{t}^{2}+0.11 c_{t}\left(c_{t}-1\right), \\
& \mu_{h \gamma \gamma} \simeq\left(1.28-0.28 c_{t}\right)^{2}+\left(0.43 \tilde{c}_{t}\right)^{2} .
\end{aligned}
$$

We perform the $\chi^{2}$ fit of anomalous couplings $c_{t}$ and $\tilde{c}_{t}$ to the Higgs data by using the package HiggsSignals-1.2.0 [107, 108].

\section{$2.1 \quad g g \rightarrow h h$}

In figure 1, we show the Feynman diagrams of the process $g g \rightarrow h h$ at the LHC. As above mentioned, the process $g g \rightarrow h h$ is generated by triangle and box top quark loop diagrams, respectively. By applying the low energy theorem, we can obtain the effective coupling of any number of neutral scalar Higgs boson to two gluons [109, 110],

$$
\mathcal{L}_{\text {hgg }}=\frac{\alpha_{s}}{12 \pi} G_{\mu \nu}^{a} G^{a \mu \nu} \log \left(1+\frac{h}{v}\right)=\frac{\alpha_{s}}{12 \pi}\left(\frac{h}{v}-\frac{h^{2}}{2 v^{2}}+\cdots\right) G_{\mu \nu}^{a} G^{a \mu \nu} .
$$

The first two interactions govern the cross section for di-Higgs production via the gluon fusion in the heavy top limit. From eq. (2.7), we can see that there is a strong cancellation between the triangle and box top quark loops diagrams because of the opposite signs of the effective couplings. So if the new physics can flip the relative sign of them, the cross section of process $g g \rightarrow h h$ may be greatly enhanced. 

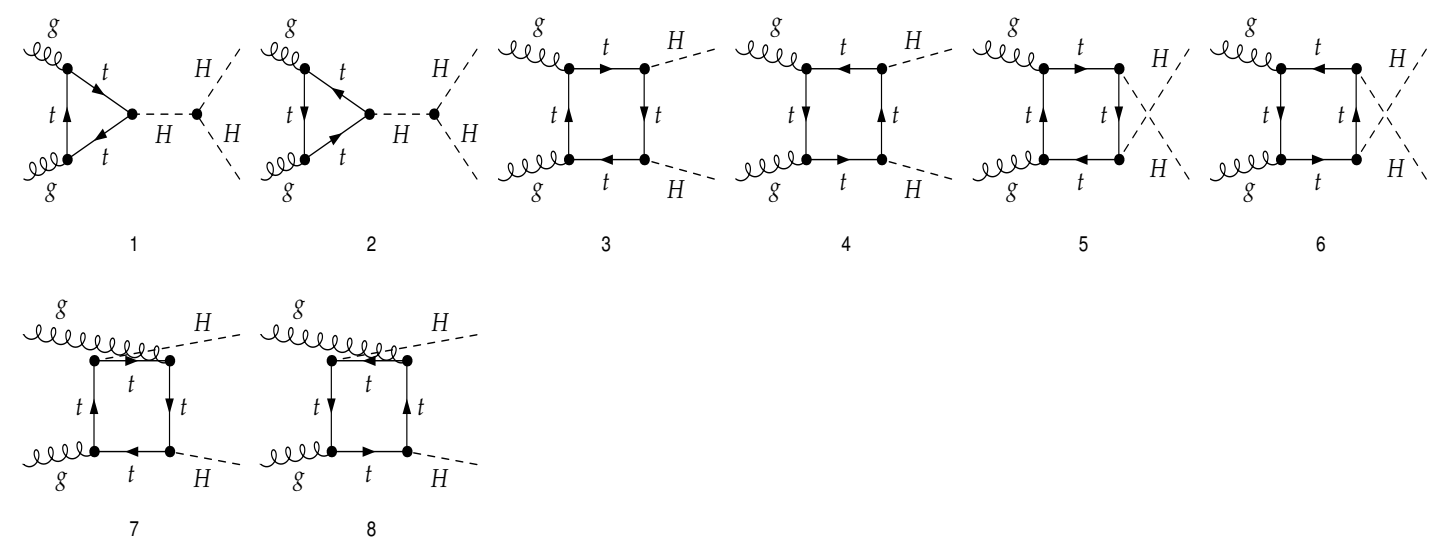

Figure 1. Feynman diagrams of the process $g g \rightarrow h h$ at the LHC.
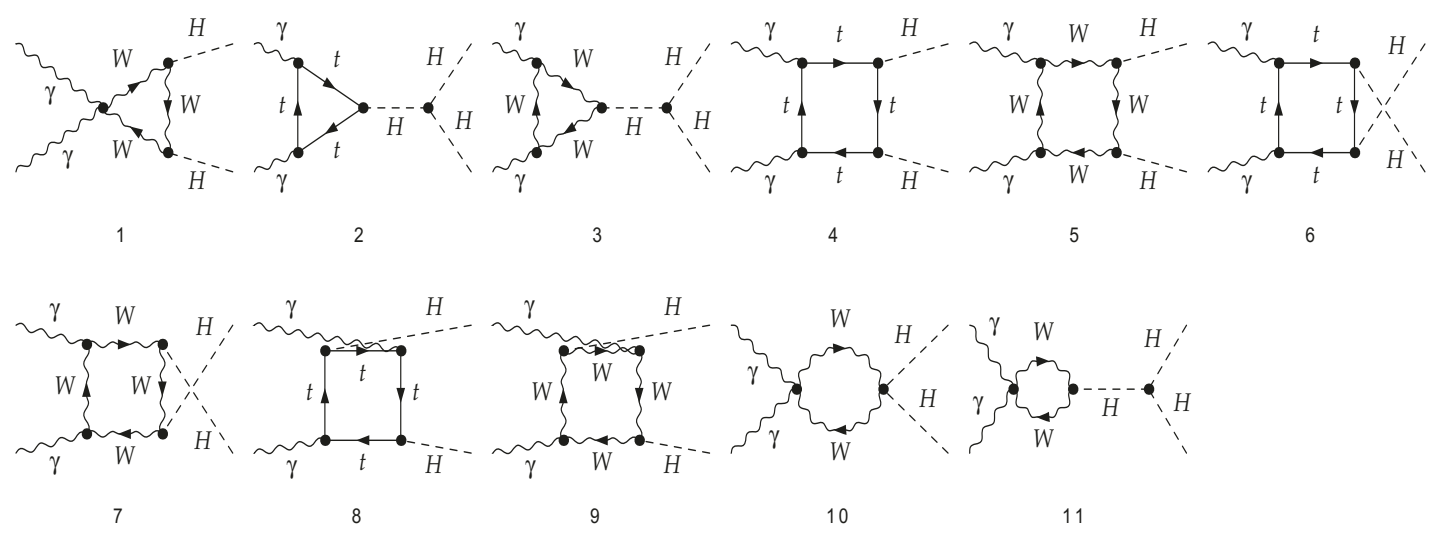

Figure 2. Feynman diagrams of the process $\gamma \gamma \rightarrow h h$ at photon collider.

\section{$2.2 \gamma \gamma \rightarrow h h$}

In figure 2, we show the Feynman diagrams of the process $\gamma \gamma \rightarrow h h$, which is governed by $W$ boson and top quark loop diagrams, respectively. At the ILC, the $\gamma \gamma$ collisions are obtained by the inverse Compton scattering of the incident electron- and the laser-beam, the events number is calculated by convoluting the cross section of $\gamma \gamma$ collision with the photon beam luminosity distribution:

$$
N_{\gamma \gamma \rightarrow h h}=\int d \sqrt{s_{\gamma \gamma}} \frac{d \mathcal{L}_{\gamma \gamma}}{d \sqrt{s_{\gamma \gamma}}} \hat{\sigma}_{\gamma \gamma \rightarrow h h}\left(s_{\gamma \gamma}\right) \equiv \mathcal{L}_{e^{+} e^{-}} \sigma_{\gamma \gamma \rightarrow h h}(s)
$$

where $d \mathcal{L}_{\gamma \gamma} / d \sqrt{s}_{\gamma \gamma}$ is the photon-beam luminosity distribution and $\sigma_{\gamma \gamma \rightarrow h h}(s)$ ( $s$ is the squared center-of-mass energy of $e^{+} e^{-}$collision) is defined as the effective cross section of $\gamma \gamma \rightarrow h h$, which can be written as $[111,112]$

$$
\sigma_{\gamma \gamma \rightarrow h h}(s)=\int_{\sqrt{a}}^{x_{\max }} 2 z d z \hat{\sigma}_{\gamma \gamma \rightarrow h h}\left(s_{\gamma \gamma}=z^{2} s\right) \int_{z^{2 / x_{\max }}}^{x_{\max }} \frac{d x}{x} F_{\gamma / e}(x) F_{\gamma / e}\left(\frac{z^{2}}{x}\right)
$$


where $F_{\gamma / e}$ denotes the energy spectrum of the back-scattered photon for the unpolarized initial electron and laser photon beams given by

$$
F_{\gamma / e}(x)=\frac{1}{D(\xi)}\left[1-x+\frac{1}{1-x}-\frac{4 x}{\xi(1-x)}+\frac{4 x^{2}}{\xi^{2}(1-x)^{2}}\right]
$$

with

$$
D(\xi)=\left(1-\frac{4}{\xi}-\frac{8}{\xi^{2}}\right) \ln (1+\xi)+\frac{1}{2}+\frac{8}{\xi}-\frac{1}{2(1+\xi)^{2}}
$$

Here $\xi=4 E_{e} E_{0} / m_{e}^{2}$ ( $E_{e}$ is the incident electron energy and $E_{0}$ is the initial laser photon energy) and $x=E / E_{0}$ with $E$ being the energy of the scattered photon moving along the initial electron direction. In the calculations, we fix the parameters as $\xi=4.8, D(\xi)=1.83$ and $x_{\max }=0.83[111,112]$.

Similar to the lagrangian $\mathcal{L}_{h g g}$, the effective coupling of any number of neutral scalar Higgs boson to two photons can be given as [110, 113],

$$
\mathcal{L}_{h \gamma \gamma}=\frac{\alpha_{e m}}{2 \pi}\left(N_{c} \frac{Q_{t}^{2}}{3}-\frac{7}{4}\right) F^{\mu \nu} F_{\mu \nu} \log \left(1+\frac{h}{v}\right)=-\frac{47 \alpha_{e m}}{72 \pi}\left(\frac{h}{v}-\frac{h^{2}}{2 v^{2}}+\cdots\right) F_{\mu \nu} F^{\mu \nu} .
$$

There is also the cancellation between the triangle and box loop diagrams. But different from $g g \rightarrow h h$, the contributions to the process $\gamma \gamma \rightarrow h h$ are dominated by the $W$ boson loops. So, the effect of the non-standard top-Higgs coupling on the di-Higgs production at photon collider may be smaller than that at the LHC.

For the loop calculations, we generate and simplify the amplitudes by using the packages FeynArts-3.9 [114] and FormCalc-8.2[115]. All the loop functions are numerically calculated with the package LoopTools-2.8 [116].

\section{3 numerical results and discussions}

In the numerical calculations, we take the input parameters of the SM as [117]

$$
\begin{aligned}
& m_{t}=173.07 \mathrm{GeV} \text {, } \\
& m_{W}=80.385, \\
& m_{Z}=91.19 \mathrm{GeV}, \\
& m_{h}=125.9 \mathrm{GeV} \text {, } \\
& \sin ^{2} \theta_{W}=0.2228 \text {, } \\
& \alpha\left(m_{Z}\right)^{-1}=127.918 \text {. }
\end{aligned}
$$

For the strong coupling constant $\alpha_{s}(\mu)$, we use its 2-loop evolution with QCD parameter $\Lambda^{n_{f}=5}=226 \mathrm{MeV}$ and get $\alpha_{s}\left(m_{Z}\right)=0.118$. We use CTEQ6M parton distribution functions (PDF) for the calculation of $g g \rightarrow h h[118]$. The renormalization scale $\mu_{R}$ and factorization scale $\mu_{F}$ are chosen to be $\mu_{R}=\mu_{F}=m_{h}$. We numerically checked that all the UV divergences in the loop corrections canceled. Since the cross section of di-Higgs production is determined by the phase angle $\theta$ and the coupling $y_{t}$, we will firstly assume $y_{t}=y_{t_{\mathrm{SM}}}$ and take $\theta=0, \pi / 2, \pi$ for example to illustrate the effects of different phase angles on the di-Higgs production at the LHC and ILC-based photon collider in figure 3 and figure 5, respectively. Then, we will vary both of $y_{t}$ and $\theta$ and respectively present the ratios of $\sigma^{g g \rightarrow h h} / \sigma_{\mathrm{SM}}^{g g \rightarrow h h}$ and $\sigma^{\gamma \gamma \rightarrow h h} / \sigma_{\mathrm{SM}}^{\gamma \gamma \rightarrow h h}$ under the constraint of the Higgs data 


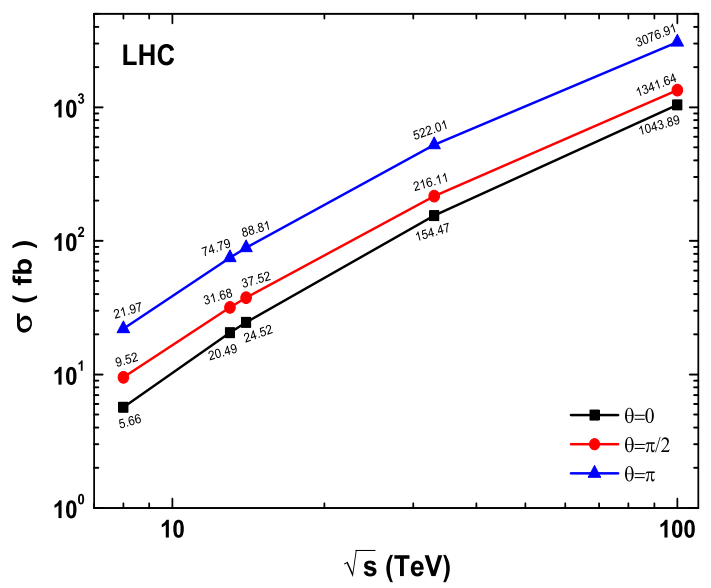

Figure 3. Cross sections of the process $g g \rightarrow h h$ for non-standard top-Higgs couplings with $y_{t}=y_{t_{\mathrm{SM}}}$ and $\theta=0, \pi / 2, \pi$ at the LHC with $\sqrt{s}=8,13,14,33,100 \mathrm{TeV}$.

in figure 4 and figure 6 . Here it should be noted that when $\theta \neq 0$, the coupling $y_{t}$ with the SM value can be potentially dangerous because such a value may violate perturbative expansion of effective field theory and/or be inconsistent with the current LHC limit on the scale of new physics. So, in that case, $y_{t}<y_{t_{\mathrm{SM}}}$ is usually needed to satisfy the theoretical and experimental bounds, which can be seen from figure 4 and figure 6 . For example, when $\theta=\pi / 4, y_{t} / y_{t_{\mathrm{SM}}}$ should be within the range $0.4-0.6$.

\section{$3.1 g g \rightarrow h h$}

In figure 3 , we present the impact of the non-standard top-Higgs couplings with $y_{t}=$ $y_{t_{\mathrm{SM}}}$ and $\theta=0, \pi / 2, \pi$ on the cross section of the di-Higgs production at the LHC with $\sqrt{s}=8,13,14,33,100 \mathrm{TeV}$. From figure 3, we can find that the top-Higgs coupling with an inverse sign $y_{t}=-y_{t_{\mathrm{SM}}}$ can significantly enhance the di-Higgs cross sections from $24.52 \mathrm{fb}$ to $88.61 \mathrm{fb}$, while the pseudo-scalar top-Higgs coupling can moderately increase the cross section up to $37.58 \mathrm{fb}$ at $\sqrt{s}=14 \mathrm{TeV}$. The reason is that these non-standard top-Higgs interactions can change the interference behavior of the triangle and box top loop diagrams in the process $g g \rightarrow h h$. To be specific, the amplitudes of these two kinds of Feynman diagrams for $\sqrt{s} \gg m_{t}, m_{h}$ in the SM can be approximately written as,

$$
\begin{aligned}
\mathcal{M}_{\text {box }}^{t} & \sim y_{t}^{2} \alpha_{s} \frac{m_{t}^{2}}{v^{2}}, \\
\mathcal{M}_{\text {triangle }}^{t} & \sim-y_{t} \lambda_{h h h} \alpha_{s} \frac{m_{t}^{2}}{v^{2}} \frac{m_{h}^{2}}{\hat{s}}\left[\log \left(\frac{m_{t}^{2}}{\hat{s}}\right)+i \pi\right]^{2} .
\end{aligned}
$$

where we take the SM Higgs self-coupling $\lambda_{h h h}=3 m_{h}^{2} / v$ in our study. For our cases, (i) when $\theta=\pi$, the top-Higgs coupling $y_{t_{\mathrm{SM}}}$ becomes $-y_{t_{\mathrm{SM}}}$ so that the sign of $\mathcal{M}_{\text {triangle }}$ is same as $\mathcal{M}_{\text {box }}$; (ii) when $\theta=\pi / 2$, the SM coupling $y_{t_{\mathrm{SM}}}$ changes to $i y_{t_{\mathrm{SM}}}$. So there is no interference between the $\mathcal{M}_{\text {triangle }}$ and $\mathcal{M}_{\text {box }}$, which will provide a constructive contribution to $g g \rightarrow h h$. 


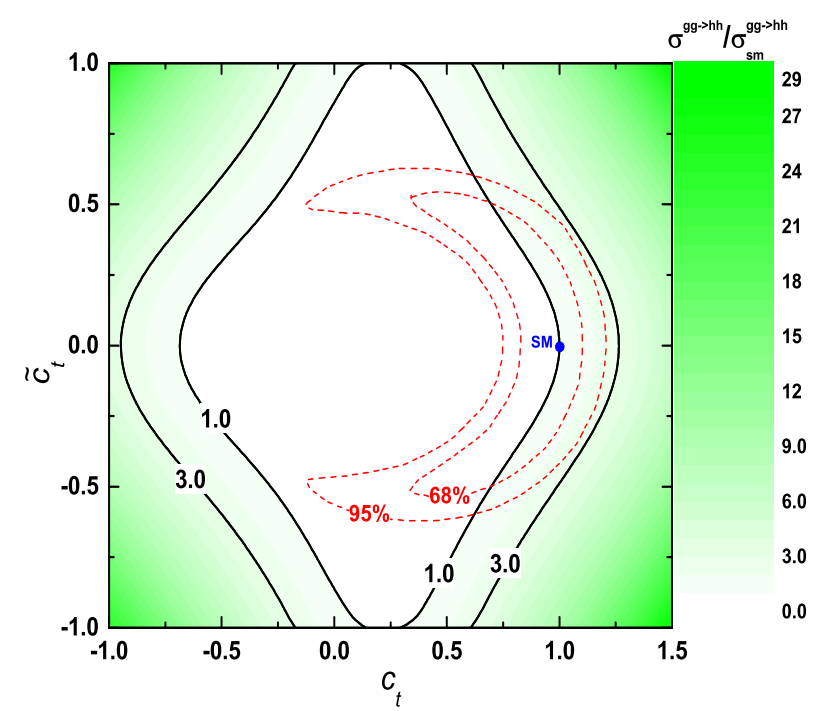

Figure 4. Ratios of $\sigma^{g g \rightarrow h h} / \sigma_{\mathrm{SM}}^{g g \rightarrow h h}$ at $14 \mathrm{TeV}$ in the plane of $\tilde{c}_{t}-c_{t}$, where the dashed contours correspond to the $68 \%$ C.L. and $95 \%$ C.L. limits given by the current Higgs data fitting.

Besides, we can see that the cross section of $g g \rightarrow h h$ becomes larger with the increase of $\sqrt{s}$ and can reach about $1 \mathrm{pb}$ in the $\mathrm{SM}$ at $\sqrt{s}=100 \mathrm{TeV}$, which is about 40 times larger than the one at $\sqrt{s}=14 \mathrm{TeV}$. However, it should be noted that the amplitudes of triangle diagrams is suppressed by center of mass energy $\hat{s}$. The box diagrams will dominate the contribution to $g g \rightarrow h h$ at the VLHC. This means that the extraction of the Higgs selfcoulping from the measurement of the total cross section of $g g \rightarrow h h$ will strongly depend on the assumption of the top-Higgs coupling at the VLHC. In this case, a study of the kinematic distributions of the Higgs bosons is needed to identify the sources of new physics in di-Higgs production.

In figure 4 , we plot the ratios of $\sigma^{g g \rightarrow h h} / \sigma_{\mathrm{SM}}^{g g \rightarrow h h}$ at $14 \mathrm{TeV}$ in the plane of $\tilde{c}_{t}-c_{t}$, where the dashed contours correspond to the $68 \%$ C.L. and $95 \%$ C.L. limits given by the current Higgs data fitting. From figure 4, we can see that the positive reduced scalar couplings $c_{t}>0.5$ are strongly favoured and the reduced pseudoscalar couplings in the range $\tilde{c}_{t}>0.6$ have been excluded at $95 \%$ C.L by the Higgs data fitting. This severely constrains the enhancement of the di-Higgs production at the LHC. So the maximal value of the ratio $\sigma^{g g \rightarrow h h} / \sigma_{\mathrm{SM}}^{g g \rightarrow h h}$ can only reach about 3 in the $95 \%$ C.L. allowed region at $14 \mathrm{TeV}$ LHC. On the other hand, the precise measurement of $g g \rightarrow h h$ will further bound these non-standard top-Higgs couplings.

\subsection{ILC-based photon collider}

In figure 5, we show the cross sections of $\gamma \gamma \rightarrow h h$ in the presence of the non-standard top-Higgs couplings with $y_{t}=y_{t_{\mathrm{SM}}}$ and $\theta=0, \pi / 2, \pi$ at the ILC-based photon collider with $\sqrt{s}=310,350,500,800,1000 \mathrm{GeV}$. From figure 5, we can see that the cross sections for pseudo-scalar coupling $\theta=\pi / 2$ is smaller than that for the SM coupling $\theta=0$. This is different from the case of $g g \rightarrow h h$ at the LHC, where only top quark propagates in the loops. But for the process $\gamma \gamma \rightarrow h h, W$ boson loops will be involved and have an 


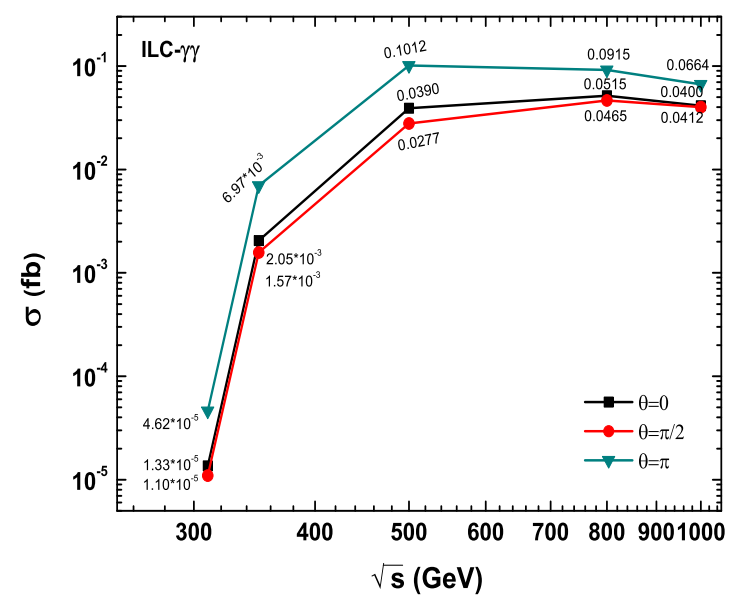

Figure 5. Cross sections of $\gamma \gamma \rightarrow h h$ in the presence of the non-standard top-Higgs couplings with $y_{t}=y_{t_{\mathrm{SM}}}$ and $\theta=0, \pi / 2, \pi$ at the ILC-based photon collider with $\sqrt{s}=310,350,500,800,1000 \mathrm{GeV}$.

interference with the top quark loops. To be specific, the amplitudes of the top quark and $W$ boson box diagrams with $\sqrt{s} \gg m_{t}, m_{W}, m_{h}$ in the SM can be approximately written as,

$$
\begin{aligned}
& \mathcal{M}_{\text {box }}^{t} \sim y_{t}^{2} Q_{t}^{2} \alpha \frac{m_{t}^{2}}{v^{2}}, \\
& \mathcal{M}_{\text {box }}^{W} \sim y_{W_{\mathrm{SM}}}^{2} Q_{W}^{2} \alpha \frac{m_{W}^{2}}{v^{2}} .
\end{aligned}
$$

where $Q_{t, W}$ is the electric charge of top quark and $W$ boson, respectively. $y_{W_{\mathrm{SM}}}=$ $g M_{Z} / \cos \theta_{W}$ denotes the SM Higgs gauge coupling and is fixed in our calculations. For $\theta=\pi / 2$, the coefficient of the amplitude of the top quark box diagrams will be changed from $y_{t_{\mathrm{SM}}}^{2}$ to $-y_{t_{\mathrm{SM}}}^{2}$. So, as comparison with the SM prediction, the relative sign between the $W$ boson box and the top quark box will be inverted, which leads to a cancellation between them; for $\theta=\pi$, the flipped sign of $y_{t_{\mathrm{SM}}}$ can increase the cross section of $\gamma \gamma \rightarrow h h$ in two sides: one is from the enhancement of those triangle diagrams involving $h \gamma \gamma$; the other one is from the constructive interference between the top quark triangle and box diagrams. We also note that for different non-standard top-Higgs couplings, the cross section of $\gamma \gamma$ always reach the maximal value at $\sqrt{s}=500 \mathrm{GeV}$. This is caused by the threshold effect of the top quark pair in the loop. When $\sqrt{s}$ becomes larger, the cross section will decrease.

Similar to figure 4, we plot the ratios of $\sigma^{\gamma \gamma \rightarrow h h} / \sigma_{\mathrm{SM}}^{\gamma \gamma \rightarrow h h}$ at ILC-based photon collider with $\sqrt{s}=500 \mathrm{GeV}$ in the plane of $\tilde{c}_{t}-c_{t}$. From figure 6 , we can see that although the cross section of $\sigma^{\gamma \gamma \rightarrow h h}$ can be about 13.9 times the SM prediction, the maximal value of the ratio $\sigma^{\gamma \gamma \rightarrow h h} / \sigma_{\mathrm{SM}}^{\gamma \gamma \rightarrow h h}$ can only reach about 2 in the region allowed by the current Higgs data at $95 \%$ C.L.. So, given the latest analysis of the feasibility of $\gamma \gamma \rightarrow h h \rightarrow b \bar{b} b \bar{b}$, such an enhancement effect can be observed at the future photon collider.

\section{Conclusions}

After LHC Run-I, measurement of Higgs self-coupling is one of the crucial tasks at future colliders, such as the LHC Run-II and the ILC-based photon collider. In this paper, 


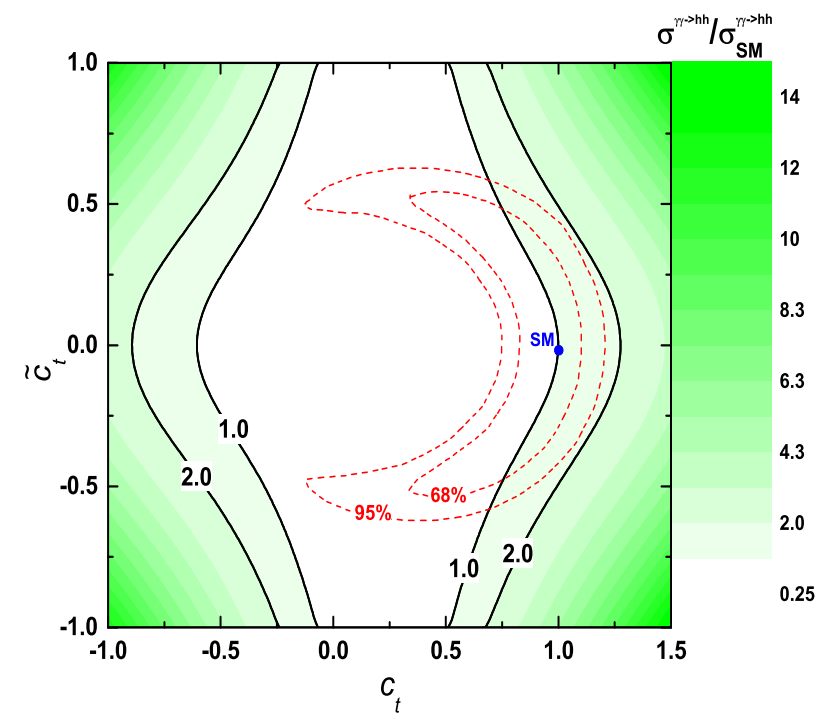

Figure 6. Similar to figure 4 , but ratios of $\sigma^{\gamma \gamma \rightarrow h h} / \sigma_{\mathrm{SM}}^{\gamma \gamma \rightarrow h h}$ at ILC-based photon collider with $\sqrt{s}=500 \mathrm{GeV}$ in the plane of $\tilde{c}_{t}-c_{t}$.

given the recent Higgs data, we investigate the di-Higgs production in the presence of the non-standard top-Higgs coupling at the LHC and ILC-based photon collider. Due to the changed interference behaviors of the top quark loops with itself or $W$ boson loops, we find that the cross section of di-Higgs production at the LHC-14 TeV and ILC-500 GeV can be respectively enhanced up to nearly 3 and 2 times the SM predictions within $2 \sigma$ Higgs data allowed parameter region.

\section{Acknowledgments}

This work is supported by the National Natural Science Foundation of China (NNSFC) under grants Nos. 11405047, 11275057, 11305049 and 11347140, by Specialised Research Fund for the Doctoral Program of Higher Education under Grant No. 20134104120002 and by the Startup Foundation for Doctors of Henan Normal University under contract No. 11112 by the China Postdoctoral Science Foundation under Grant No. 2014M561987 and the Joint Funds of the National Natural Science Foundation of China (U1404113).

Open Access. This article is distributed under the terms of the Creative Commons Attribution License (CC-BY 4.0), which permits any use, distribution and reproduction in any medium, provided the original author(s) and source are credited.

\section{References}

[1] ATLAS collaboration, Observation of a new particle in the search for the standard model Higgs boson with the ATLAS detector at the LHC, Phys. Lett. B 716 (2012) 1 [arXiv:1207.7214] [INSPIRE].

[2] CMS collaboration, Observation of a new boson at a mass of $125 \mathrm{GeV}$ with the CMS experiment at the LHC, Phys. Lett. B 716 (2012) 30 [arXiv:1207.7235] [INSPIRE]. 
[3] ATLAS collaboration, Measurements of the properties of the Higgs-like boson in the two photon decay channel with the ATLAS detector using $25 \mathrm{fb}^{-1}$ of proton-proton collision data, ATLAS-CONF-2013-012 (2013).

[4] ATLAS collaboration, Combined coupling measurements of the Higgs-like boson with the ATLAS detector using up to $25 \mathrm{fb}^{-1}$ of proton-proton collision data, ATLAS-CONF-2013-034 (2013).

[5] CMS collaboration, Updated measurements of the Higgs boson at $125 \mathrm{GeV}$ in the two photon decay channel, CMS-PAS-HIG-13-001 (2013).

[6] CMS collaboration, Combination of standard model Higgs boson searches and measurements of the properties of the new boson with a mass near $125 \mathrm{GeV}$, CMS-PAS-HIG-13-005 (2013).

[7] ATLAS collaboration, Search for the standard model Higgs boson produced in association with top quarks and decaying to $b \bar{b}$ in pp collisions at $\sqrt{s}=8 \mathrm{TeV}$ with the ATLAS detector at the LHC, ATLAS-CONF-2014-011 (2014).

[8] CMS collaboration, Search for $t t H$ production using the Matrix Element Method, CMS-PAS-HIG-14-010 (2014).

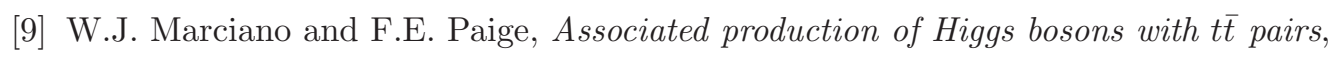
Phys. Rev. Lett. 66 (1991) 2433 [INSPIRE].

[10] J. Dai, J.F. Gunion and R. Vega, Using $b$ tagging to detect $t \bar{t}$ Higgs production with Higgs $\rightarrow b \bar{b}$, Phys. Rev. Lett. 71 (1993) 2699 [hep-ph/9306271] [INSPIRE].

[11] J. Goldstein et al., $p \bar{p} \rightarrow t \bar{t} H:$ a discovery mode for the Higgs boson at the Tevatron, Phys. Rev. Lett. 86 (2001) 1694 [hep-ph/0006311] [INSPIRE].

[12] A. Belyaev and L. Reina, $p p \rightarrow t \bar{t} H, H \rightarrow \tau^{+} \tau^{-}$: toward a model independent determination of the Higgs boson couplings at the LHC, JHEP 08 (2002) 041 [hep-ph/0205270] [INSPIRE].

[13] F. Maltoni, D.L. Rainwater and S. Willenbrock, Measuring the top quark Yukawa coupling at hadron colliders via $t \bar{t} H, H \rightarrow W^{+} W^{-}$, Phys. Rev. D 66 (2002) 034022 [hep-ph/0202205] [INSPIRE].

[14] V. Drollinger, T. Müller and D. Denegri, Searching for Higgs bosons in association with top quark pairs in the $H^{0} \rightarrow b \bar{b}$ decay mode, hep-ph/0111312 [INSPIRE].

[15] W. Beenakker et al., NLO QCD corrections to $t \bar{t} H$ production in hadron collisions, Nucl. Phys. B 653 (2003) 151 [hep-ph/0211352] [InSPIRE].

[16] P. Agrawal, S. Bandyopadhyay and S.P. Das, Multilepton signatures of the Higgs boson through its production in association with a top-quark pair, Phys. Rev. D 88 (2013) 093008 [arXiv: 1308.3043] [INSPIRE].

[17] S. Biswas, R. Frederix, E. Gabrielli and B. Mele, Enhancing the t $\bar{t} H$ signal through top-quark spin polarization effects at the LHC, JHEP 07 (2014) 020 [arXiv:1403.1790] [INSPIRE].

[18] M.V. Garzelli, A. Kardos, C.G. Papadopoulos and Z. Trócsányi, Standard model Higgs boson production in association with a top anti-top pair at NLO with parton showering, Europhys. Lett. 96 (2011) 11001 [arXiv:1108.0387] [INSPIRE].

[19] R. Frederix et al., Scalar and pseudoscalar Higgs production in association with a top-antitop pair, Phys. Lett. B 701 (2011) 427 [arXiv:1104.5613] [INSPIRE].

[20] C. Degrande, J.M. Gerard, C. Grojean, F. Maltoni and G. Servant, Probing top-Higgs non-standard interactions at the LHC, JHEP 07 (2012) 036 [Erratum ibid. 1303 (2013) 032] [arXiv:1205.1065] [INSPIRE]. 


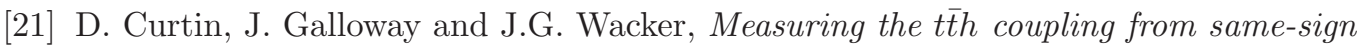
dilepton +2b measurements, Phys. Rev. D 88 (2013) 093006 [arXiv:1306.5695] [InSPIRE].

[22] J. Adelman, A. Loginov, P. Tipton and J. Vasquez, Study of $t \bar{t} H\left(H \rightarrow \mu^{+} \bar{\mu}^{-}\right)$in the three lepton channel at $\sqrt{s}=14 \mathrm{TeV}$; a Snowmass white paper, arXiv:1310.1132 [INSPIRE].

[23] CMS Collaboration, Search for associated production of a single top quark and a Higgs boson in events where the Higgs boson decays to two photons at $\sqrt{s}=8 \mathrm{TeV}$, CMS-PAS-HIG-14-001 (2014).

[24] G. Bordes and B. van Eijk, On the associate production of a neutral intermediate mass Higgs boson with a single top quark at the LHC and SSC, Phys. Lett. B 299 (1993) 315 [INSPIRE].

[25] A. Ballestrero and E. Maina, $t \bar{b} H$ production for an intermediate mass Higgs, Phys. Lett. B 299 (1993) 312 [INSPIRE].

[26] W.J. Stirling and D.J. Summers, Production of an intermediate mass Higgs boson in association with a single top quark at LHC and SSC, Phys. Lett. B 283 (1992) 411 [INSPIRE].

[27] J.L. Diaz-Cruz and O.A. Sampayo, Associated production of the Higgs boson with $t \bar{b}$ at hadron colliders, Phys. Lett. B 276 (1992) 211 [INSPIRE].

[28] T.M.P. Tait and C.-P. Yuan, Single top quark production as a window to physics beyond the standard model, Phys. Rev. D 63 (2000) 014018 [hep-ph/0007298] [INSPIRE].

[29] F. Maltoni, K. Paul, T. Stelzer and S. Willenbrock, Associated production of Higgs and single top at hadron colliders, Phys. Rev. D 64 (2001) 094023 [hep-ph/0106293] [INSPIRE].

[30] P. Agrawal, S. Mitra and A. Shivaji, Effect of anomalous couplings on the associated production of a single top quark and a Higgs boson at the LHC, JHEP 12 (2013) 077 [arXiv:1211.4362] [INSPIRE].

[31] Y. Wang et al., Constraints on flavor-changing neutral-current Htq couplings from the signal of $t H$ associated production with QCD next-to-leading order accuracy at the LHC, Phys. Rev. D 86 (2012) 094014 [arXiv: 1208.2902] [INSPIRE].

[32] J. Cao, Z. Heng, L. Wu and J.M. Yang, Top quark forward-backward asymmetry at the Tevatron: a comparative study in different new physics models, Phys. Rev. D 81 (2010) 014016 [arXiv:0912.1447] [InSPIRE].

[33] M. Farina, C. Grojean, F. Maltoni, E. Salvioni and A. Thamm, Lifting degeneracies in Higgs couplings using single top production in association with a Higgs boson, JHEP 05 (2013) 022 [arXiv:1211.3736] [InSPIRE].

[34] S. Biswas, E. Gabrielli and B. Mele, Single top and Higgs associated production as a probe of the Htt coupling sign at the LHC, JHEP 01 (2013) 088 [arXiv: 1211.0499] [INSPIRE].

[35] J. Ellis, D.S. Hwang, K. Sakurai and M. Takeuchi, Disentangling Higgs-top couplings in associated production, JHEP 04 (2014) 004 [arXiv: 1312.5736] [INSPIRE].

[36] C. Englert and E. Re, Bounding the top Yukawa coupling with Higgs-associated single-top production, Phys. Rev. D 89 (2014) 073020 [arXiv: 1402.0445] [INSPIRE].

[37] J. Chang, K. Cheung, J.S. Lee and C.-T. Lu, Probing the top-yukawa coupling in associated Higgs production with a single top quark, JHEP 05 (2014) 062 [arXiv:1403.2053] [INSPIRE].

[38] A. Kobakhidze, L. Wu and J. Yue, Anomalous top-Higgs couplings and top polarisation in single top and higgs associated production at the LHC, JHEP 10 (2014) 100 [arXiv: 1406.1961] [INSPIRE]. 
[39] L. Wu, Enhancing thj Production from top-higgs FCNC couplings, arXiv:1407.6113 [INSPIRE].

[40] M. Gillioz, R. Grober, C. Grojean, M. Muhlleitner and E. Salvioni, Higgs low-energy theorem (and its corrections) in composite models, JHEP 10 (2012) 004 [arXiv:1206.7120] [INSPIRE].

[41] R. Contino et al., Anomalous couplings in double Higgs production, JHEP 08 (2012) 154 [arXiv: 1205.5444] [INSPIRE].

[42] S. Dawson, E. Furlan and I. Lewis, Unravelling an extended quark sector through multiple Higgs production?, Phys. Rev. D 87 (2013) 014007 [arXiv:1210.6663] [INSPIRE].

[43] G. Bhattacharyya, D. Das and P.B. Pal, Modified Higgs couplings and unitarity violation, Phys. Rev. D 87 (2013) 011702 [arXiv:1212.4651] [INSPIRE].

[44] C. Han, A. Kobakhidze, N. Liu, L. Wu and B. Yang, Constraining top partner and naturalness at the LHC and TLEP, Nucl. Phys. B 890 (2015) 388 [arXiv:1405.1498] [INSPIRE].

[45] K. Nishiwaki, S. Niyogi and A. Shivaji, ttH anomalous coupling in double Higgs production, JHEP 04 (2014) 011 [arXiv: 1309.6907] [InSPIRE].

[46] C.-R. Chen and I. Low, Double take on new physics in double Higgs boson production, Phys. Rev. D 90 (2014) 013018 [arXiv:1405.7040] [INSPIRE].

[47] C.-Y. Chen, S. Dawson and I.M. Lewis, Top partners and Higgs boson production, Phys. Rev. D 90 (2014) 035016 [arXiv:1406.3349] [inSPIRE].

[48] W. Hollik and S. Penaranda, Yukawa coupling quantum corrections to the selfcouplings of the lightest MSSM Higgs boson, Eur. Phys. J. C 23 (2002) 163 [hep-ph/0108245] [INSPIRE].

[49] A. Dobado, M.J. Herrero, W. Hollik and S. Penaranda, Selfinteractions of the lightest MSSM Higgs boson in the large pseudoscalar mass limit, Phys. Rev. D 66 (2002) 095016 [hep-ph/0208014] [INSPIRE].

[50] M. Brucherseifer, R. Gavin and M. Spira, MSSM Higgs self-couplings: two-loop $\mathcal{O}\left(\alpha_{t} \alpha_{s}\right)$ corrections, arXiv:1309.3140 [INSPIRE].

[51] S. Kanemura, S. Kiyoura, Y. Okada, E. Senaha and C.P. Yuan, New physics effect on the Higgs selfcoupling, Phys. Lett. B 558 (2003) 157 [hep-ph/0211308] [INSPIRE].

[52] S. Kanemura, Y. Okada, E. Senaha and C.-P. Yuan, Higgs coupling constants as a probe of new physics, Phys. Rev. D 70 (2004) 115002 [hep-ph/0408364] [INSPIRE].

[53] V. Barger, L.L. Everett, C.B. Jackson and G. Shaughnessy, Higgs-pair production and measurement of the triscalar coupling at LHC(8,14), Phys. Lett. B 728 (2014) 433 [arXiv:1311.2931] [INSPIRE].

[54] V. Barger, L.L. Everett, C.B. Jackson, A.D. Peterson and G. Shaughnessy, Measuring the 2HDM scalar potential at LHC14, Phys. Rev. D 90 (2014) 095006 [arXiv:1408.2525] [INSPIRE].

[55] R. Grober and M. Muhlleitner, Composite Higgs boson pair production at the LHC, JHEP 06 (2011) 020 [arXiv: 1012.1562] [INSPIRE].

[56] R.S. Gupta, H. Rzehak and J.D. Wells, How well do we need to measure the Higgs boson mass and self-coupling?, Phys. Rev. D 88 (2013) 055024 [arXiv: 1305.6397] [INSPIRE].

[57] A. Efrati and Y. Nir, What if $\lambda_{h h h} \neq 3 m_{h}^{2} / v$, arXiv:1401.0935 [INSPIRE]. 
[58] S. Kanemura, Y. Okada and E. Senaha, Electroweak baryogenesis and quantum corrections to the triple Higgs boson coupling, Phys. Lett. B 606 (2005) 361 [hep-ph/0411354] [INSPIRE].

[59] S. Kanemura, E. Senaha, T. Shindou and T. Yamada, Electroweak phase transition and Higgs boson couplings in the model based on supersymmetric strong dynamics, JHEP 05 (2013) 066 [arXiv:1211.5883] [INSPIRE].

[60] D.Y. Shao, C.S. Li, H.T. Li and J. Wang, Threshold resummation effects in Higgs boson pair production at the LHC, JHEP 07 (2013) 169 [arXiv:1301.1245] [INSPIRE].

[61] D. de Florian and J. Mazzitelli, Higgs boson pair production at next-to-next-to-leading order in QCD, Phys. Rev. Lett. 111 (2013) 201801 [arXiv:1309.6594] [INSPIRE].

[62] X. Li and M.B. Voloshin, Remarks on double Higgs boson production by gluon fusion at threshold, Phys. Rev. D 89 (2014) 013012 [arXiv:1311.5156] [INSPIRE].

[63] R. Frederix et al., Higgs pair production at the LHC with NLO and parton-shower effects, Phys. Lett. B 732 (2014) 142 [arXiv:1401.7340] [INSPIRE].

[64] J. Grigo, K. Melnikov and M. Steinhauser, Virtual corrections to Higgs boson pair production in the large top quark mass limit, Nucl. Phys. B 888 (2014) 17 [arXiv: 1408.2422] [INSPIRE].

[65] N.D. Christensen, T. Han and T. Li, Pair production of MSSM Higgs bosons in the non-decoupling region at the LHC, Phys. Rev. D 86 (2012) 074003 [arXiv:1206.5816] [INSPIRE].

[66] J. Cao, Z. Heng, L. Shang, P. Wan and J.M. Yang, Pair production of a $125 \mathrm{GeV}$ Higgs boson in MSSM and NMSSM at the LHC, JHEP 04 (2013) 134 [arXiv:1301.6437] [INSPIRE].

[67] U. Ellwanger, Higgs pair production in the NMSSM at the LHC, JHEP 08 (2013) 077 [arXiv: 1306.5541] [INSPIRE].

[68] C. Han, X. Ji, L. Wu, P. Wu and J.M. Yang, Higgs pair production with SUSY QCD correction: revisited under current experimental constraints, JHEP 04 (2014) 003 [arXiv: 1307.3790] [INSPIRE].

[69] B. Bhattacherjee and A. Choudhury, The role of MSSM heavy Higgs production in the self coupling measurement of $125 \mathrm{GeV}$ Higgs boson at the LHC, arXiv:1407.6866 [INSPIRE].

[70] N. Chen et al., LHC searches for the heavy Higgs boson via two B jets plus diphoton, Phys. Rev. D 89 (2014) 115006 [arXiv:1312.7212] [INSPIRE].

[71] J. Baglio, O. Eberhardt, U. Nierste and M. Wiebusch, Benchmarks for Higgs pair production and heavy Higgs boson searches in the two-Higgs-doublet model of type II, Phys. Rev. D 90 (2014) 015008 [arXiv:1403.1264] [InSPIRE].

[72] B. Hespel, D. Lopez-Val and E. Vryonidou, Higgs pair production via gluon fusion in the two-Higgs-doublet model, JHEP 09 (2014) 124 [arXiv: 1407.0281] [INSPIRE].

[73] V. Barger, L.L. Everett, C.B. Jackson, A. Peterson and G. Shaughnessy, New physics in resonant production of Higgs boson pairs, arXiv: 1408.0003 [INSPIRE].

[74] G.D. Kribs and A. Martin, Enhanced di-Higgs production through light colored scalars, Phys. Rev. D 86 (2012) 095023 [arXiv:1207.4496] [INSPIRE].

[75] M.J. Dolan, C. Englert and M. Spannowsky, New physics in LHC Higgs boson pair production, Phys. Rev. D 87 (2013) 055002 [arXiv:1210.8166] [INSPIRE]. 
[76] H. Sun and Y.-J. Zhou, Enhancement of loop induced $125 \mathrm{GeV}$ Higgs pair production through large-extra-dimensions model at the LHC, JHEP 11 (2012) 127 [arXiv:1211.6201] [INSPIRE].

[77] J. Guo, Z. Kang, J. Li and T. Li, Implications of Higgs sterility for the Higgs and stop sectors, arXiv: 1308.3075 [INSPIRE].

[78] J. Liu, X.-P. Wang and S.-h. Zhu, Discovering extra Higgs boson via pair production of the SM-like Higgs bosons, arXiv:1310.3634 [INSPIRE].

[79] J.M. No and M. Ramsey-Musolf, Probing the Higgs portal at the LHC through resonant di-Higgs production, Phys. Rev. D 89 (2014) 095031 [arXiv:1310.6035] [INSPIRE].

[80] N. Haba, K. Kaneta, Y. Mimura and E. Tsedenbaljir, Higgs pair production at the LHC and ILC from general potential, Phys. Rev. D 89 (2014) 015018 [arXiv:1311.0067] [INSPIRE].

[81] Z. Heng, L. Shang, Y. Zhang and J. Zhu, Pair production of $125 \mathrm{GeV}$ Higgs boson in the SM extension with color-octet scalars at the LHC, JHEP 02 (2014) 083 [arXiv:1312.4260] [INSPIRE].

[82] Q. Li, Q.-S. Yan and X. Zhao, Higgs pair production: improved description by matrix element matching, Phys. Rev. D 89 (2014) 033015 [arXiv:1312.3830] [INSPIRE].

[83] T. Enkhbat, Scalar leptoquarks and Higgs pair production at the LHC, JHEP 01 (2014) 158 [arXiv: 1311.4445] [INSPIRE].

[84] T. Li, Decoupling MSSM Higgs sector and heavy Higgs decay, Phys. Lett. B 728 (2014) 77 [arXiv: 1309.6713] [INSPIRE].

[85] A.J. Barr, M.J. Dolan, C. Englert and M. Spannowsky, Di-Higgs final states augMT2ed Selecting hh events at the high luminosity LHC, Phys. Lett. B 728 (2014) 308 [arXiv:1309.6318] [INSPIRE].

[86] U. Baur, T. Plehn and D.L. Rainwater, Determining the Higgs boson selfcoupling at hadron colliders, Phys. Rev. D 67 (2003) 033003 [hep-ph/0211224] [INSPIRE].

[87] U. Baur, T. Plehn and D.L. Rainwater, Probing the Higgs selfcoupling at hadron colliders using rare decays, Phys. Rev. D 69 (2004) 053004 [hep-ph/0310056] [INSPIRE].

[88] M.J. Dolan, C. Englert and M. Spannowsky, Higgs self-coupling measurements at the LHC, JHEP 10 (2012) 112 [arXiv:1206.5001] [INSPIRE].

[89] J. Baglio et al., The measurement of the Higgs self-coupling at the LHC: theoretical status, JHEP 04 (2013) 151 [arXiv: 1212.5581] [INSPIRE].

[90] A. Papaefstathiou, L.L. Yang and J. Zurita, Higgs boson pair production at the LHC in the $b \bar{b} W^{+} W^{-}$channel, Phys. Rev. D 87 (2013) 011301 [arXiv:1209.1489] [INSPIRE].

[91] M.J. Dolan, C. Englert, N. Greiner and M. Spannowsky, Further on up the road: hhjj production at the LHC, Phys. Rev. Lett. 112 (2014) 101802 [arXiv:1310.1084] [INSPIRE].

[92] P. Maierhöfer and A. Papaefstathiou, Higgs boson pair production merged to one jet, JHEP 03 (2014) 126 [arXiv:1401.0007] [INSPIRE].

[93] D.E. Ferreira de Lima, A. Papaefstathiou and M. Spannowsky, Standard model Higgs boson pair production in the $(b \bar{b})(b \bar{b})$ final state, JHEP 08 (2014) 030 [arXiv:1404.7139] [INSPIRE].

[94] F. Goertz, A. Papaefstathiou, L.L. Yang and J. Zurita, Higgs boson self-coupling measurements using ratios of cross sections, JHEP 06 (2013) 016 [arXiv:1301.3492] [INSPIRE].

[95] D.M. Asner et al., ILC Higgs white paper, arXiv:1310.0763 [INSPIRE]. 
[96] I.F. Ginzburg, G.L. Kotkin, V.G. Serbo and V.I. Telnov, Production of high-energy colliding $\gamma \gamma$ and $\gamma$ e beams with a high luminosity at Vlepp accelerators, JETP Lett. 34 (1981) 491 [Pisma Zh. Eksp. Teor. Fiz. 34 (1981) 514] [InSPIRE].

[97] R. Belusevic and G. Jikia, Higgs selfcoupling in $\gamma \gamma$ collisions, Phys. Rev. D 70 (2004) 073017 [hep-ph/0403303] [INSPIRE].

[98] E. Asakawa, D. Harada, S. Kanemura, Y. Okada and K. Tsumura, Higgs boson pair production at a photon-photon collision in the two Higgs doublet model, Phys. Lett. B 672 (2009) 354 [arXiv:0809.0094] [InSPIRE].

[99] E. Asakawa, D. Harada, S. Kanemura, Y. Okada and K. Tsumura, Higgs boson pair production in new physics models at hadron, lepton and photon colliders, Phys. Rev. D 82 (2010) 115002 [arXiv:1009.4670] [INSPIRE].

[100] Z. Heng, L. Shang and P. Wan, Pair production of a $125 \mathrm{GeV}$ Higgs boson in MSSM and NMSSM at the ILC, JHEP 10 (2013) 047 [arXiv: 1306.0279] [INSPIRE].

[101] I. Ginzburg and M. Krawczyk, Testing Higgs physics at the photon collider, arXiv: 1310.5881 [INSPIRE].

[102] S.-i. Kawada et al., A feasibility study of the measurement of Higgs pair creation at a photon linear collider, Phys. Rev. D 85 (2012) 113009 [arXiv: 1205.5292] [INSPIRE].

[103] J.A. Aguilar-Saavedra, A Minimal set of top-Higgs anomalous couplings, Nucl. Phys. B 821 (2009) 215 [arXiv:0904.2387] [INSPIRE].

[104] D. Atwood, S. Bar-Shalom, G. Eilam and A. Soni, CP violation in top physics, Phys. Rept. 347 (2001) 1 [hep-ph/0006032] [INSPIRE].

[105] A. Kobakhidze, Standard Model with a distorted Higgs sector and the enhanced Higgs diphoton decay rate, arXiv:1208.5180 [INSPIRE].

[106] J. Brod, U. Haisch and J. Zupan, Constraints on CP-violating Higgs couplings to the third generation, JHEP 11 (2013) 180 [arXiv:1310.1385] [INSPIRE].

[107] P. Bechtle et al., HiggsSignals: confronting arbitrary Higgs sectors with measurements at the Tevatron and the LHC, Eur. Phys. J. C 74 (2014) 2711 [arXiv:1305.1933] [INSPIRE].

[108] P. Bechtle, O. Brein, S. Heinemeyer, G. Weiglein and K.E. Williams, HiggsBounds: confronting arbitrary Higgs sectors with exclusion bounds from LEP and the Tevatron, Comput. Phys. Commun. 181 (2010) 138 [arXiv:0811.4169] [INSPIRE].

[109] K. Hagiwara and H. Murayama, Multiple weak boson production via gluon fusion, Phys. Rev. D 41 (1990) 1001 [INSPIRE].

[110] B.A. Kniehl and M. Spira, Low-energy theorems in Higgs physics, Z. Phys. C 69 (1995) 77 [hep-ph/9505225] [INSPIRE].

[111] I.F. Ginzburg et al., Colliding $\gamma e$ and $\gamma \gamma$ beams based on single-pass $e^{+} e^{-}$accelerators II. Polarization effects, monochromatization improvement,

Nucl. Instrum. Meth. A 219 (1984) 5.

[112] V.I. Telnov, Problems in obtaining $\gamma \gamma$ and $\gamma$ e colliding beams at linear colliders, Nucl. Instrum. Meth. A 294 (1990) 72.

[113] M.A. Shifman, A.I. Vainshtein, M.B. Voloshin and V.I. Zakharov, Low-energy theorems for Higgs boson couplings to photons, Sov. J. Nucl. Phys. 30 (1979) 711 [Yad. Fiz. 30 (1979) 1368] [INSPIRE].

[114] T. Hahn, Generating Feynman diagrams and amplitudes with FeynArts 3, Comput. Phys. Commun. 140 (2001) 418 [hep-ph/0012260] [INSPIRE]. 
[115] T. Hahn and M. Pérez-Victoria, Automatized one loop calculations in four-dimensions and D-dimensions, Comput. Phys. Commun. 118 (1999) 153 [hep-ph/9807565] [INSPIRE].

[116] G.J. van Oldenborgh, $F F-A$ package to evaluate one-loop Feynman diagrams, Comput. Phys. Commun. 66 (1991) 1.

[117] Particle Data Group collaboration, J. Beringer et al., Review of particle physics, Phys. Rev. D 86 (2012) 010001 [inSPIRE].

[118] P.M. Nadolsky et al., Implications of CTEQ global analysis for collider observables, Phys. Rev. D 78 (2008) 013004 [arXiv: 0802.0007] [INSPIRE]. 